\title{
ARTICLE OPEN \\ Inhomogeneous strain-induced half-metallicity in bent zigzag graphene nanoribbons
}

\author{
Dong-Bo Zhang ${ }^{1}$ and Su-Huai Wei ${ }^{1}$
}

Realization of half-metallicity in low dimensional materials is a fundamental challenge for nano spintronics, which is a critical component for next-generation information technology. Using the method of generalized Bloch theorem, we show that an in-plane bending can induce inhomogeneous strains, which in turn lead to spin-splitting in zigzag graphene nanoribbons and results in the highly desired half-metallic state. Unlike the previously proposed scheme that requires unrealistically strong external electric fields, the obtained half-metallicity with sizeable half-metallic gap and high energetic stability of magnetic order of edge states requires only relatively low-level strain in the in-plane bending. Given the superior structural flexibility of graphene and the recent experimental advances in controllable synthesis of graphene nanoribbons, our design provides a hitherto most practical approach to the realization of half-metallicity in low dimensional systems. This work, thus paves a way towards the design of nanoscale spintronic devices through strain engineering.

npj Computational Materials (2017)3:32; doi:10.1038/s41524-017-0036-9

\section{INTRODUCTION}

Having one spin channel conducting while the other one insulating, half-metallicity (HM) can provide completely spinpolarized charge carriers. This exotic property represents an ideal condition for spintronics which manipulates the spin freedom of electrons for various applications such as logic circuits, data storage and information processing. ${ }^{1-4}$ Since the first theoretical prediction, ${ }^{5}$ great efforts have been devoted to identify or design materials with $\mathrm{HM}$ properties. ${ }^{6-14}$ So far, HM has been experimentally demonstrated in bulk systems, e.g., ferromagnetic manganese perovskite. ${ }^{6}$ However, despite great efforts, ${ }^{7-14}$ the actual realization of $\mathrm{HM}$ in low dimensional materials, especially in two-dimensional (2D) single atomic/molecular layer, has not been realized. This has hindered design of spintronic devices at nanoscale.

Graphene has been expected to be the long-awaited platform for $2 D$ spintronics ${ }^{15}, 16$ given its unique electronic properties such as extremely long spin diffusion length due to its massless Dirac Fermion nature ${ }^{17}$ and the weak spin-orbit interaction. ${ }^{18}$ More importantly, graphene is predicted to have spin-polarized edge states with a sizeable band gap ${ }^{19}$ when graphene is cut into zigzag terminated graphene nanoribbons (GNR). Figure 1a showcases a zigzag GNR with $n=8 \mathrm{C}$ chains. It has a band structure with a finite bandgap, where the valance band (VB) and conduction band (CB) consist of localized edge states at $2 \pi / 3<$ $k \mathbf{T}<4 \pi / 3{ }^{7}$ where $\mathbf{T}$ represents the translational vector, see Fig. $2 \mathrm{~b}$. These edge states are intrinsically spin-polarized with ferromagnetic order for one edge of the GNR (but being anti-ferromagnetic (AFM) for the opposite sides) without involving any transition metal elements for both VB, Fig. 2d, and CB, Fig. 2e. Given such a special spin configuration, it is possible to induce spin-splitting, e.g., by exerting an electric field transversely across the GNR. In this case, the energy levels of the localized edge states on the opposite edges will shift relatively with respect to each other for both spin orientations. ${ }^{7}$

Son et al. first suggested that this spin-splitting might result in a HM state under an electric field with proper strength, and demonstrated their proposal with first-principles calculations. ${ }^{7}$ However, the required electric fields are too strong to reach at such nanoscale given the state-of-art gate technique. ${ }^{20}$ It has also been suggested that the required electric field can be alternatively obtained via chemical decoration of GNR edges with selective atoms or functional groups. ${ }^{8,20-24}$ Although the resulting $\mathrm{HM}$ is indeed pronounced, it is impractical to accomplish the controllable doping of atoms/functional groups at the GNR edges with atomic precision. Recently, efforts were also devoted to graphene/ hexagonal boron nitrogen $(h B N)$ lateral heterostructures where a zigzag GNR is sandwiched by $h \mathrm{BN}$ NRs with one side terminated by $\mathrm{B}$ and the other side by $\mathrm{N}$ in order to build intrinsically an electric field. ${ }^{25-27}$ However, the obtained $\mathrm{HM}$ are found to be severely suppressed with a vanishingly small HM gap. ${ }^{28}$

Here we reveal a new mechanism to realize the HM states in zigzag GNRs by in-plane bending, where GNRs are bent along the axial direction as shown in Fig. 1b. This concept is inspired by an unusual phenomenon uncovered in zigzag GNRs that the edge states response opposed to tension and compression deformations. This, together with the spatial separation of edge states between opposite spin orientations, paves an alternative way to induce spin-splitting in edge states and consequently, provides an opportunity to achieve the HM state by deforming zigzag GNRs inhomogeneously with proper strain pattern. We find that a simple in-plane bending can fulfill this requirement. As an illustration, we carried out systematic electronic band structure calculations of bent zigzag GNRs. Such simulation is possible only due to our recent theoretical advance of the development of the generalized Bloch theorem coupled with self-consistent charge density-functional tight-binding (SCC-DFTB). The outcomes totally

${ }^{1}$ Beijing Computational Science Research Center, Beijing 100193, P. R. China

Correspondence: Dong-Bo Zhang (dbzhang@csrc.ac.cn) or Su-Huai Wei (suhuaiwei@csrc.ac.cn)

Received: 19 April 2017 Revised: 29 June 2017 Accepted: 14 July 2017

Published online: 14 August 2017 


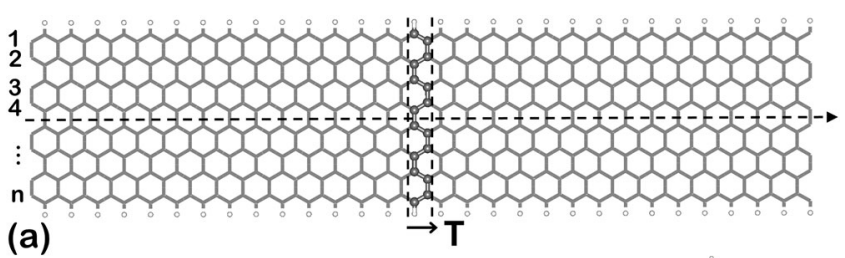

(a)

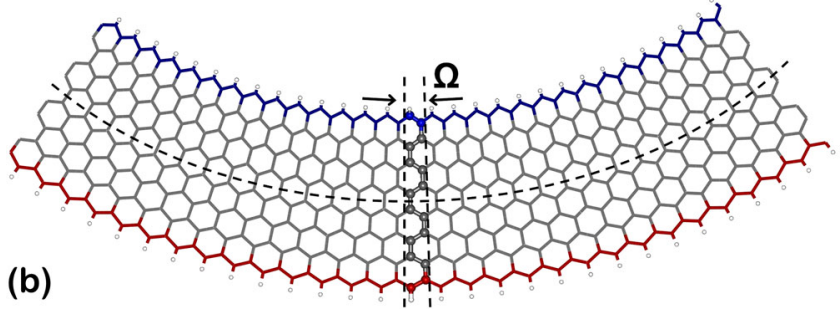

Fig. 1 a A zigzag GNR with $n=8 \mathrm{C}$ chains with hydrogen saturated on both edges. The GNR is infinite long along its axis as indicated by the dashed arrow. $\mathbf{T}$ represents the translational vector. Those $16 \mathrm{C}$ atoms inside the translational unit cell are represented by solid balls. b The same zigzag GNR under a uniform in-plane bending. The rotational angle corresponding to this motif is $\Omega$. The dashed line indicates the neutral surface with zero strain. On the inner (outer) side of the neutral surface, the C chain colored blue (red) along GNR edge is compressed (extended) (a)
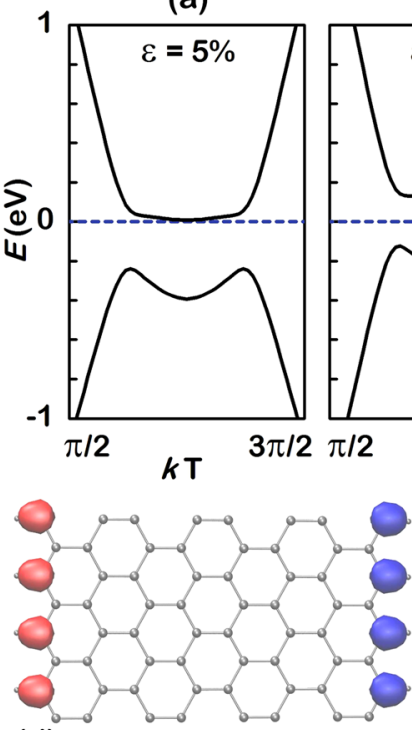

(d) (b)

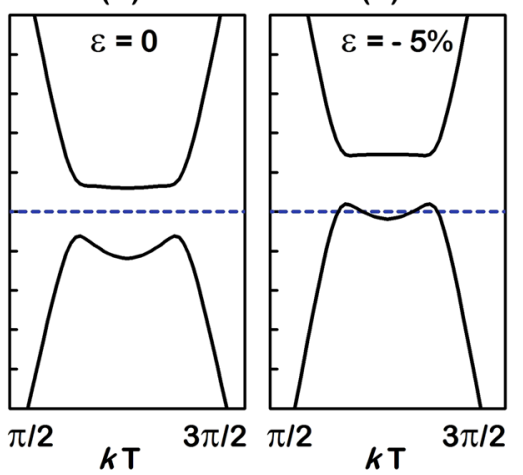

Fig. 2 Electronic band structures of a zigzag GNR with $n=8 \mathrm{C}$ chains under different conditions: a axial tension $\varepsilon=5 \%$, b stressfree $\varepsilon=0$, and $c$ axial compression $\varepsilon=-5 \%$. The spatial distribution of the spin density of the edge states for the same zigzag GNR are given in $\mathbf{d}$ for VB edge state and $\mathbf{e}$ CB edge state. The red and blue colors represent the spin-up and spin-down orientations, respectively. The isosurface level of charge densities is $0.01 \mathrm{e} / \AA$

support our expectation. The well-defined HM state with a sizeable HM gap is achieved while the excellent stability of the long-range spin order ${ }^{7}$ in zigzag GNRs is well retained under this in-plane bending. The maximum strains involved in the needed bending deformations are only at mediate level ( 10\%) for all the GNRs considered. Given the superior structural flexibility of graphene, ${ }^{29}$ our design is promising for a practical realization given the present experimental advances in the fabrication of GNR samples with sharp zigzag edges ${ }^{30,} 31$ and in controlling the interplay between GNRs and substrates. ${ }^{32}$

\section{RESULTS}

Strain effects on edges states

We first examine the strain effect on edge states by carrying out electronic band structure calculation using SCC-DFTB method. ${ }^{33}$ Considering tension and compression, we find that energy levels of both VB and CB of edge states shift downwards and upwards with respect to the stress-free case for zigzag GNRs under tension and compression, respectively, as shown in Fig. 2a, c. A parallel calculation using the first-principle pseudopotential VASP code $^{34}$ for the same zigzag GNR shows very similar results (see Supplementary Note 1). To characterize the dependence of edge states on strain, systematic studies are conducted for a strain range of $-5 \%<\varepsilon<5 \%$. Results are shown in Supplementary Note 2. Our calculation reveals that the energy level shifts of edge states with $\varepsilon$ are nearly linear. We note that the bandgap is almost impervious to strain because the energies of the VB and $C B$ of edge states move in the same direction. This is quite unusual since in conventional covalent semiconductors band gaps usually changes linearly and sensitively with strain, where the energies of the band edge states move in opposite directions.

This interesting phenomenon can be explained by noticing that the spin-polarization-induced bandgap has VB and CB states with similar character and the variation of kinetic energy of these edge states under strains is responsible for the energy levels shift. As an example, we focus on edge states located at the boundary of Brillouin zone, i.e., $K=\pi /|\mathbf{T}|$. At strain $\varepsilon$, the translational periodicity, $|\mathbf{T}|=(1+\varepsilon)\left|\mathbf{T}_{0}\right|$. Here, $\mathbf{T}_{0}$ represents the translational vector of the stress-free zigzag GNR. The kinetic energy of the edge states is approximately,

$$
\begin{aligned}
E_{K} & =\frac{\hbar^{2} K^{2}}{2 m_{e}^{*}} \\
& =\frac{\hbar^{2} \pi^{2}}{2 m_{e}^{*}\left|T_{0}\right|^{2}} \frac{1}{(1+\varepsilon)^{2}} \\
& \cong a(1-2 \varepsilon),
\end{aligned}
$$

where, $m_{e}^{*}$ and $\hbar$ are effective electron mass and Plank constant, respectively, and $a=\hbar^{2} \pi^{2} /\left(2 m_{e}^{*}\left|\mathbf{T}_{0}\right|^{2}\right)$. Equation (1) reveals that the energy of edge states increases (decreases) with compression (tension) nearly linearly, consistent with our band structure calculation. We indicate that the uncovered distinct responses of edge states to tension and compression, combining the spatial separation of edge states between opposite spin orientations as shown in Fig. 2d, e, can be utilized to induce spin-splitting in edge states, and may likely lead to the desired HM state by deforming zigzag GNRs inhomogeneously with proper strain pattern. In the present work, we choose a simple in-plane bending.

\section{Bending induced spin splitting and HM states}

Our main results are summarized in Fig. 3. As expected, for a zigzag GNR with $n=8 \mathrm{C}$ chains, there is a spin-splitting of edge states which increases with the bending angle $\Omega$, where the $C B$ (VB) of spin-up states shifts downwards (upwards), while the $C B$ (VB) of spin-down states shifts upwards (downwards), see for example Fig. 3b. Figure 3d plots the dependence of band gaps on $\Omega$ for both spin-up and spin-down states, which is almost linear as function of $\Omega$. We can see that, at $\Omega=2.5^{\circ}$, the gap between the $\mathrm{CB}$ and VB of spin-up states reaches a complete closure, indicating the emergence of HM state, Fig. 3c. At this critical bending angle, the bandgap between the $C B$ and $V B$ of spin-down states is usually referred as the HM gap. Here, it is $\sim 0.39 \mathrm{eV}$ for GNR with 
(a)
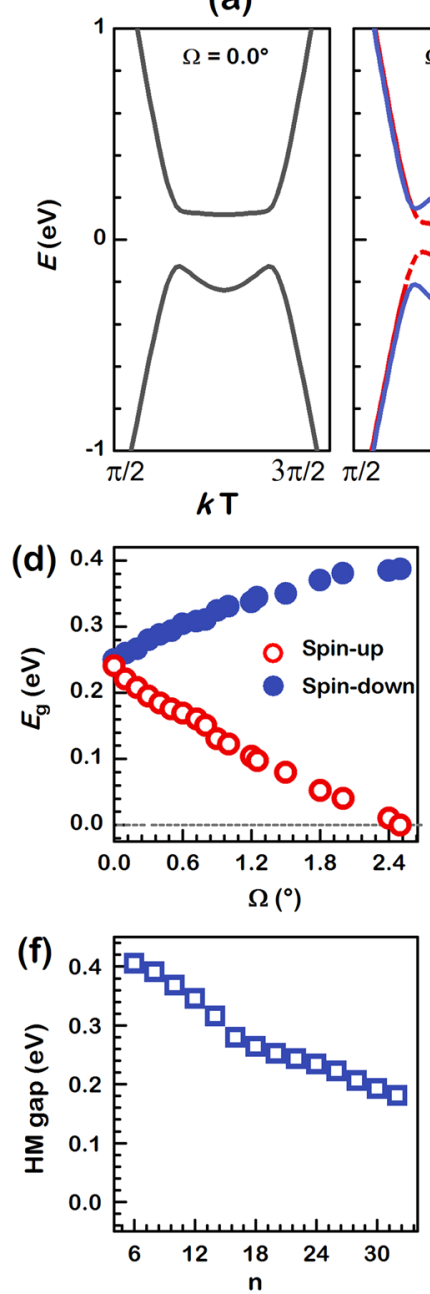

(b)

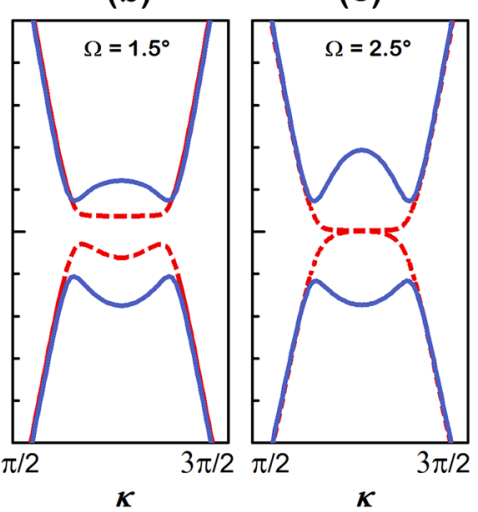

(e)
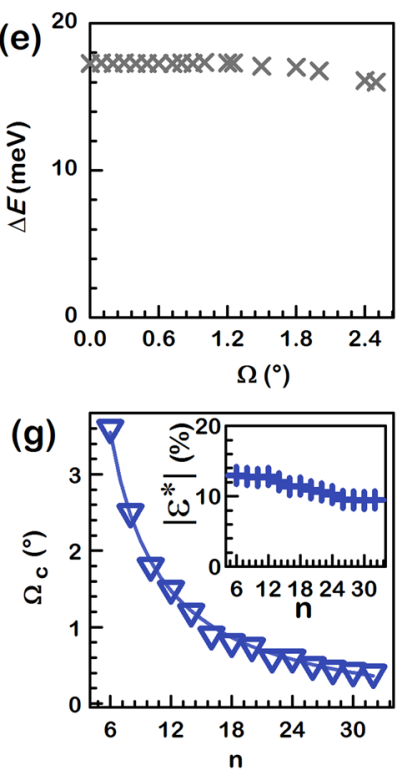

Fig. 3 Spin-resolved electronic band structures of a zigzag GNR with $n=8 \mathrm{C}$ chains for the stress-free case $\mathbf{a}$, and under in-plane bending with different bending angles $(\Omega): 1.5^{\circ} \mathbf{b}$, and $2.5^{\circ} \mathbf{c}$. The red dashed and blue solid lines denote bands of spin-up states and spin-down states, respectively. The Fermi energy is set to zero. $\mathbf{d}$ Dependence of band gaps on bending angle $\Omega$ for spin-up states (red opened circle) and spin-down states (blue filled square). e Dependence of magnetic interaction energy $\Delta \mathrm{E}$ per edge $\mathrm{C}$ atom on bending angle $\Omega$. $\mathbf{f}$ Dependence of the HM gap induced by bending as a function of the number of $C$ chains $n$ of the zigzag GNRs. $g$ Critical bending angle $\Omega_{c}$ to induce the HM states as a function of the number of $C$ chains $n$ of the zigzag GNRs. The solid curve indicates the reversely proportional dependence of $\Omega_{c}$ on $n$. The insert shows the corresponding strains at GNR edges at these critical bending angles

$n=8 \mathrm{C}$ chains. This is important because to maintain high degree of spin-polarization in a spintronic application, large HM gap is required.

For a practical useful HM system, not only the sizeable HM gap is needed, the stability of the long-range magnetic order is also a necessity. To verify the magnetic stability, we calculate the energetic difference $\Delta E=E_{N M}-E_{A F M}$ between the AFM and nonmagnetic states (NM), which is often used to characterize the magnetic stability of a system. ${ }^{7}$ Here, $E_{A F M}$ and $E_{N M}$ denote the energies of the AFM and NM states, respectively. For stressfree zigzag GNRs $\left(\Omega=0.0^{\circ}\right)$, the magnetic interaction energy is indeed large. ${ }^{7}$ For example, for the zigzag GNR with $n=8 \mathrm{C}$ chains, $\Delta \mathrm{E} \approx 17.5 \mathrm{meV}$ per edge $\mathrm{C}$ atom given by SCC-DFTB. Under

bending, the variation of $\Delta \mathrm{E}$ is negligible as shown in Fig. $3 \mathrm{e}$, suggesting that the magnetic stability of zigzag GNRs is well preserved.

We further examine the size dependence of the bending induced HM. Figure $3 f$ shows that the HM gap decreases slowly with GNR width but remains sizeable with values larger than 0.2 $\mathrm{eV}$ when the number of $\mathrm{C}$ chains in GNR increases from $n=6$ to 32 , indicating that the $\mathrm{HM}$ is pronounced for a wide range of GNR widths, see Supplementary Note 3 for more details. We have also determined the critical bending angle $\Omega_{c}$ for each considered $\mathrm{GNR}$, at which the HM state emerges. Figure $3 \mathrm{~g}$ shows that a smaller bending angle $\Omega_{\mathrm{c}}$ is needed to approach the HM state as the GNR becomes wider. Our SCC-DFTB calculation finds a simple relationship $\Omega_{c}=8.9\left(n W_{0} / \AA\right)^{-1}$, where $W_{0}=2.14 \AA$ is the unit width along the width dimension of GNR, revealing that $\Omega_{c}$ decreases inversely proportionally with GNR width $n W_{0}$, as shown in Fig. 3g. This trend can be understood as follows: In our approach, the strain is applied through in-plane bending. In this case, the outer side (inner side) edge of the GNR is extended (compressed) with $\varepsilon$ given as

$\varepsilon= \pm \frac{n \mathrm{~W}_{0} \Omega}{\left|\mathbf{T}_{0}\right|}$

The insert of Fig. $3 g$ shows that the critical strain $\left|\varepsilon^{*}\right|$, which triggers the $\mathrm{HM}$ has only a weak size dependence with values varying $\sim 10 \%$, thus, from Eq. (2), we can derive that $\Omega_{c}$ is inversely proportional to $n$. Given the superior structural flexibility of graphene, ${ }^{29}$ such strain level is expected to be easily achievable. For example, the in-plane bending can be applied in a similar manner as in several previous studies, ${ }^{35}, 36$ where graphene samples are placed on some flexible substrates, see Fig. 4 for a schematic setup. For the maintenance of the one-dimensional spin-polarized edge states of GNRs, strong electronic interaction between GNRs and the substrate should be avoided. We thus suggest that certain polymer insulators such as polyethylene terephthalate film and poly-methyl methacrylate can be used, which lock the graphene piece essentially through non-electronic van der Walls interaction. ${ }^{36,37}$

\section{DISCUSSION}

We have proposed a new mechanism to induce spin-splitting in zigzag GNRs. Using the method of generalized Bloch theorem, we show that by introducing inhomogeneous strain through in-plane bending, the HM can be achieved with a sizeable HM gap and excellent energetic stability of the magnetic order of edge states in zigzag GNRs. It is important to point out that to develop the HM through the in-plane bending, only relatively low-level strain is required. This feature indicates that our proposal is promising to realize HM in 2D systems. Our work, thus sheds lights on the new design principles of spintronic devices at nanoscale based on graphene and other 2D materials.

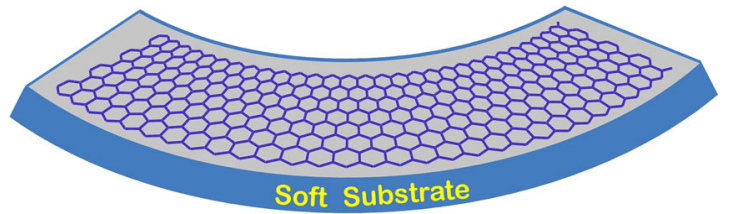

Fig. 4 Schematic representation of a possible setup for a zigzag GNR placed on certain flexible substrate. The in-plane bending is posed through the substrate 


\section{METHODS}

Because a simple bending breaks the translational symmetry, standard quantum mechanical calculations relying on the translational symmetry are not suitable any more. Thus, the present study is carried out within the theoretical framework called generalized Bloch theorem. ${ }^{38}$ In the following, we will describe some salient features of this approach: For the zigzag GNR shown in Fig. 1a, its atomic structure can be described by repeating the translational $\mathbf{T}$ vector over an $N$ atoms unit cell along its axis. However, when an in-plane bending is applied as shown in Fig. 1b, the translation symmetry is removed. Here, we instead make recourse of rotational symmetry, through which the bent GNR is described with repetition rules involving rotation $\mathbf{R}$ of angle $\Omega$ performed around the bending axis on the same $N$ atoms cell,

$\mathbf{X}_{\lambda, n}=\mathbf{R}^{\lambda}(\Omega) \mathbf{X}_{0, n}$,

where, $\mathbf{X}_{0, n}$ represents atoms inside the primitive repeating motif and $\mathbf{X}_{\lambda, n}$ represents the atoms inside the replica of the repeating motif indexed by $\lambda$. Index $n$ runs over the $N$ atoms inside the motif. The bending angle $\Omega$ of the rotational matrix $\mathbf{R}$ satisfies: $\zeta \Omega=2 \pi$ with $\zeta$ being an integer number and $\lambda$ $=0,1, \ldots, \zeta-1$. The generalized Bloch functions is represented in local basis,

$\Psi_{n, a}(\kappa, \mathbf{r})=\frac{1}{\sqrt{\zeta}} \sum_{\lambda=0}^{\zeta-1} \exp (i \kappa \lambda) \mathbf{R}^{\lambda} \varphi_{n, a}(\mathbf{r}) X(\sigma)$

where, $\phi_{n, a}(\mathbf{r})$ refers to the atomic orbital $a$ on atom $n$ and $\mathbf{R}^{\lambda} \varphi_{n, a}(\mathbf{r})$ is the symmetry-adapted orbital. ${ }^{39-41} a$ runs over all the orbitals of single atom and $n$ runs over all the $N$ atoms inside the primitive motif. The phase factors are the eigenvalues of rotation operators. Quantum number $0 \leq \kappa<$ $2 \pi$ adopts values being integral multiple of $\Omega$. $X$ represents the wavefunction for spin part with orientation $\sigma$ in the collinear treatment.

The above outlined theoretical framework has been implemented recently to the computational package dftb+ (ref. 42) by us, where the electronic band structure is calculated with SCC-DFTB. With it, we investigate a large set of zigzag GNRs with number of $C$ chains from $n=$ 6 to 32 , corresponding to a width range from 1.2 to $6.3 \mathrm{~nm}$. GNRs with greater widths are not considered here since their spin configuration becomes either ferromagnetic or not correlated between the edge states on opposite GNR edges. ${ }^{31}$ All the GNRs are hydrogen saturated at the edges. For each GNR, a series of bending angles $\Omega$ are considered, and at each $\Omega$, the atomic structure is fully relaxed via conjugate gradient energy minimization, followed by an electronic band structure calculation. $100 \mathrm{~K}$ points are used to converge the band energy.

Data availability

The authors declare that the data supporting the findings of this study are available within the paper and its Supplementary Information files.

\section{ACKNOWLEDGEMENTS}

This work was supported by the National Natural Science Foundation of China (NSFC) under Grants No. U1530401, and No. 11674022. Computations were carried out at Beijing Computational Science Research Center.

\section{AUTHOR CONTRIBUTIONS}

D.-B.Z and S.-H.W. conceived the idea and D.-B.Z. performed the calculations and the data analysis. D.-B.Z and S.-H.W. discussed the results and co-wrote the paper.

\section{ADDITIONAL INFORMATION}

Supplementary Information accompanies the paper on the npj Computational Materials website (doi:10.1038/s41524-017-0036-9).

Competing Interests: The authors declare that they have no competing financial interests.

Publisher's note: Springer Nature remains neutral with regard to jurisdictional claims in published maps and institutional affiliations.

\section{REFERENCES}

1. Wolf, S. A. et al. Spintronics: a spin-based electronics vision for the future. Science 294, 1488-1495 (2001).

2. Awschalom, D. D. \& Flatte, M. E. Challenges for semiconductor spintronics. Nat. Phys. 3, 153-159 (2007).

3. Fang, C. M., De Wijs, G. A. \& Groot, D. R. Spin-polarization in half-metals (invited). J. Appl. Phys. 91, 8340-8344 (2002).

4. Felser, C., Fecher, G. H. \& Balke, B. Spintronics: a challenge for materials science and solid-state chemistry. Angew. Chem. Int. Engl. Ed. 46, 668-699 (2007).

5. de Groot, R. A., Mueller, F. M., Engen, P. Gv \& Buschow, K. H. J. New class of materials: Half-metallic ferromagnets. Phys. Rev. Lett. 50, 2024-2027 (1983).

6. Park, J. et al. Direct evidence for a half-metallic ferromagnet. Nature 392, 794-796 (1998).

7. Son, Y., Cohen, M. L. \& Louie, S. G. Half-metallic graphene nanoribbons. Nature 444, 347-349 (2006).

8. Kan, E.-J., Li, Z., Yang, J. \& Hou, J. G. Half-metallicity in edge-modified zigzag graphene nanoribbons. J. Am. Chem. Soc. 130, 4224-4225 (2008).

9. Zhou, J. \& Sun, Q. Magnetism of phthalocyanine-based organometallic single porous sheet. J. Am. Chem. Soc. 133, 15113-15119 (2011).

10. Liu, J., Sun, Q., Kawazoe, Y. \& Jena, P. Exfoliating biocompatible ferromagnetic Crtrihalide monolayers. Phys. Chem. Chem. Phys. 18, 8777-8784 (2016).

11. Du, A., Sanvito, S. \& Smith, S. C. First-principles prediction of metal-free magnetism and intrinsic half-metallicity in graphitic carbon nitride. Phys. Rev. Lett. 108, 197207 (2012).

12. Li, X., Wu, X. \& Yang, J. Half-metallicity in $\mathrm{mnpse}_{3}$ exfoliated nanosheet with carrier doping. J. Am. Chem. Soc. 136, 11065-11069 (2014).

13. Chen, W. et al. Hydrogenation: a simple approach to realize semiconductor-halfmetal-metal transition in boron nitride nanoribbons. J. Am. Chem. Soc. 132, 1699-1705 (2010).

14. Cao, T., Li, Z. \& Louie, S. G. Tunable magnetism and half-metallicity in hole-doped monolayer GaSe. Phys. Rev. Lett. 114, 236602 (2015).

15. Han, W., Kawakami, R. K., Gmitra, M. \& Fabian, J. Graphene spintronics. Nat. Nano 9, 794-807 (2014).

16. Pesin, D. \& MacDonald, A. H. Spintronics and pseudospintronics in graphene and topological insulators. Nat. Mater. 11, 409-416 (2012).

17. Novoselov, K. S. et al. Two-dimensional gas of massless Dirac fermions in graphene. Nature 438, 197-200 (2005).

18. Kane, C. L. \& Mele, E. J. Quantum spin hall effect in graphene. Phys. Rev. Lett. 95, 226801 (2005).

19. Son, Y.-W., Cohen, M. L. \& Louie, S. G. Energy gaps in graphene nanoribbons. Phys. Rev. Lett. 97, 216803 (2006).

20. Kan, E.-J., Li, Z., Yang, J. \& Hou, J. G. Will zigzag graphene nanoribbon turn to half metal under electric field. Appl. Phys. Lett. 91, 243116 (2007).

21. Gunlycke, D., Li, J., Mintmire, J. W. \& White, C. T. Altering low-bias transport in zigzag-edge graphene nanostrips with edge chemistry. Appl. Phys. Lett. 91, 112108 (2007).

22. Hod, O., Barone, V., Peralta, J. E. \& Scuseria, G. E. Enhanced half-metallicity in edge-oxidized zigzag graphene nanoribbons. Nano Lett. 7, 2295-2299 (2007).

23. Dutta, S., Manna, A. K. \& Pati, S. K. Intrinsic half-metallicity in modified graphene nanoribbons. Phys. Rev. Lett. 102, 096601 (2009).

24. Li, Y., Zhou, Z., Shen, P. \& Chen, Z. Spin gapless semiconductor-metal-half-metal properties in nitrogen-doped zigzag graphene nanoribbons. ACS Nano 3, 1952-1958 (2009).

25. Zhang, D. et al. Interface engineering of electronic properties of graphene/boron nitride lateral heterostructures. 2D Mater. 2, 041001 (2015).

26. Pruneda, J. M. Origin of half-semimetallicity induced at interfaces of C-BN heterostructures. Phys. Rev. B 81, 161409 (2010).

27. Bhowmick, S., Singh, A. K. \& Yakobson, B. I. Quantum dots and nanoroads of graphene embedded in hexagonal boron nitride. J. Phys. Chem. C 115, 9889-9893 (2011).

28. Kim, S.-W., Kim, H.-J., Choi, J.-H., Scheicher, R. H. \& Cho, J.-H. Contrasting interedge superexchange interactions of graphene nanoribbons embedded in $h-\mathrm{BN}$ and graphane. Phys. Rev. B 92, 035443 (2015).

29. Lee, C., Wei, X., Kysar, J. W. \& Hone, J. Measurement of the elastic properties and intrinsic strength of monolayer graphene. Science 321, 385-388 (2008).

30. Ruffieux, P. et al. On-surface synthesis of graphene nanoribbons with zigzag edge topology. Nature 531, 489-492 (2016).

31. Magda, G. Z. et al. Room-temperature magnetic order on zigzag edges of narrow graphene nanoribbons. Nature 514, 608-611 (2014).

32. Wang, S. et al. Giant edge state splitting at atomically precise graphene zigzag edges. Nat. Commun. 7, 11507 (2016).

33. Elstner, M. et al. Self-consistent-charge density-functional tight-binding method for simulations of complex materials properties. Phys. Rev. B 58, 7260-7268 (1998). 
34. Kresse, G. \& Furthmüller, J. Efficient iterative schemes for ab initio total-energy calculations using a plane-wave basis set. Phys. Rev. B 54, 11169-11186 (1996).

35. Guinea, F., Geim, A. K., Katsnelson, M. I. \& Novoselov, K. S. Generating quantizing pseudomagnetic fields by bending graphene ribbons. Phys. Rev. B 81, 035408 (2010).

36. Mohiuddin, T. M. G. et al. Uniaxial strain in graphene by Raman spectroscopy: $G$ peak splitting, Grüneisen parameters, and sample orientation. Phys. Rev. B 79, 205433 (2009).

37. Tsoukleri, G. et al. Subjecting a graphene monolayer to tension and compression. Small 5, 2397-2402 (2009).

38. Sandratskii, L. M. Energy band structure calculations for crystals with spiral magnetic structure. Phys. Status Solidi B 136, 167-180 (1986).

39. White, C. T., Robertson, D. H. \& Mintmire, J. W. Helical and rotational symmetries of nanoscale graphitic tubules. Phys. Rev. B 47, 5485-5488 (1993).

40. Popov, V. N. Curvature effects on the structural, electronic and optical properties of isolated single-walled carbon nanotubes within a symmetry-adapted nonorthogonal tight-binding model. New. J. Phys. 6, 17-17 (2004).

41. Allen, P. B. Nanocrystalline nanowires: III. electrons. Nano Lett. 7, 1220-1223 (2007).
42. Aradi, B., Hourahine, B. \& Frauenheim, T. DFTB+, a sparse matrixbased implementation of the DFTB method. J. Phys. Chem. A. 111, 5678-5684 (2007).

(i) Open Access This article is licensed under a Creative Commons Attribution 4.0 International License, which permits use, sharing, adaptation, distribution and reproduction in any medium or format, as long as you give appropriate credit to the original author(s) and the source, provide a link to the Creative Commons license, and indicate if changes were made. The images or other third party material in this article are included in the article's Creative Commons license, unless indicated otherwise in a credit line to the material. If material is not included in the article's Creative Commons license and your intended use is not permitted by statutory regulation or exceeds the permitted use, you will need to obtain permission directly from the copyright holder. To view a copy of this license, visit http://creativecommons. org/licenses/by/4.0/.

C The Author(s) 2017 